\title{
Applications and limitations of micro-XCT imaging in the studies of Permian radiolarians: A new genus with bi-polar main spines
}

\author{
Yifan Xiao, Noritoshi Suzuki, and Weihong He \\ Acta Palaeontologica Polonica 62 (3), 2017: 647-656 doi:https://doi.org/10.4202/app.00367.2017
}

Microfocus X-ray Computed Tomography (micro-XCT) has been employed recently in radiolarian studies, though so far primarily to generate high quality tomographic images. Although micro-XCT technique cannot always produce high-quality tomographic images, it frequently can provide valuable information on the internal structure of spongy polycystines. Here we employ micro-XCT to understand internal skeletal structures of several Permian specimens of polycystine radiolarians. Structural inferences from micro-XCT images are compared to images of the same specimens made with SEM and transmitted light microscopy (TLM). The utility of micro-XCT for imaging internal structures is first confirmed by examining the spongy, flat, four-spined species Tetraspongodiscus stauracanthus. Micro-XCT method is then used to examine the internal structures of a spherical to elliptical polycystine with two bi-polar main spines, Dalongicaepa bipolaris Xiao and Suzuki gen. et sp. nov., from the Dalong Formation (Changhsingian) of South China. The new genus is characterized by four to seven densely concentric shells with a large spherical hollow in the center and two cylindrical spines at both poles of the cortical shell, and belongs to the family Spongotortilispinidae. Spherical to elliptical polycystines with bi-polar main spines are similar in external appearance, and their phylogenetic relationships are only determinable by examination of the internal structures. We therefore analyzed all Permian and Mesozoic spherical to elliptical polycystines with bi-polar main spines showing internal structures, using cluster analysis to measure similarity. The results show distinctive differences in internal structures and suggest that family level relationships should be revised in the future.

Key words: Radiolaria, taxonomy, micro-XCT, Permian, Changhsingian, China.

Yifan Xiao [yifanxiao@cug.edu.cn], State Key Laboratory of Biogeology and Environmental Geology, School of Earth Science, China University of Geosciences, Wuhan 430074, China; Department of Earth Science, Graduate School of Science, Tohoku University, Sendai 980-8578, Japan. Noritoshi Suzuki [norinori@m.tohoku.ac.jp], Department of Earth Science, Graduate School of Science, Tohoku University, Sendai 980-8578, Japan. Weihong He [whzhang@cug.edu.cn] (corresponding author), State Key Laboratory of Biogeology and Environmental Geology, School of 
Earth Science, China University of Geosciences, Wuhan 430074, China.

This is an open-access article distributed under the terms of the Creative Commons Attribution License (for details please see creativecommons.org), which permits unrestricted use, distribution, and reproduction in any medium, provided the original author and source are credited.

\footnotetext{
FoF Full text $(476.7 \mathrm{kB})$ ।

FoF Supplementary file $(75.2 \mathrm{kB})$
} 